\title{
Low-frequency ionisation of excited hydrogen atoms: the Floquet picture
}

\author{
H P Breuer, K Dietz and M Holthaus \\ Universität Bonn, Physikalisches Institut, Nussallee 12, D-5300 Bonn 1, West Germany
}

Received 29 March 1989, in final form 12 June 1989

\begin{abstract}
The general significance of adiabatic motion on quasi-energy surfaces for microwave ionisation of excited hydrogen atoms is investigated. It is shown that non-monotonic structures in the ionisation signal can be traced to near-degeneracies of coarse-grained quasi-energy surfaces of a few effective states. Further experimental implications are discussed.
\end{abstract}

\section{Introduction}

Experiments on microwave ionisation of highly excited hydrogen atoms (Bayfield and Koch 1974, Jensen et al 1988) can be performed under well defined conditions and therefore offer a unique opportunity to study the dynamics of periodically driven quantum systems in the case where the strength of the external driving field is of the same order of magnitude as that of the binding field. A vast number of theoretical papers (e.g. Blümel and Smilansky 1987, Casati et al 1987, Richards 1987, Jensen et al 1988) have been devoted to quite different aspects of this problem.

Recently, we have pointed out the importance of adiabatic processes: a hydrogen atom prepared in a Rydberg state crosses, when entering the microwave cavity, a fringe field where it is adiabatically shifted into the connected Floquet state (Breuer and Holthaus 1989).

In this paper, we focus on the region of low scaled frequencies $n^{3} \omega<0.1$. We point out that the current notion of adiabaticity valid in this region is contained as a limiting case in the Floquet picture $(\$ 2)$ and then present model calculations to show that coarse-graining of quasi-energy surfaces (Breuer et al 1989) yields a very simple way to understand the non-monotonic structures observed in low-frequency ionisation experiments $(\S 3)$. The last section, finally, contains our conclusions.

\section{Adiabatic evolution on effective quasi-energy surfaces}

The Schrödinger equation for a hydrogen atom in a monochromatic microwave field of strength $\lambda$ and frequency $\omega$ reads

$$
\left(H_{0}+\lambda D \sin \omega t-\mathrm{i}_{t}\right) \psi(\boldsymbol{x}, t)=0
$$

where $H_{0}$ denotes the Hamiltonian of the static atom and $D$ the dipole operator. According to Floquet's theorem (Zel'dovich 1967), there exists a complete set of 
solutions

$$
\psi_{n}(x, t)=u_{n}(x, t) \exp \left(-\mathrm{i} \varepsilon_{n} t\right)
$$

which can be obtained from the eigenvalue equation

$$
\left(H_{0}+\lambda D \sin \omega t-\mathrm{i} \partial_{t}\right) u_{\alpha}(x, t)=\varepsilon_{\alpha} u_{\alpha}(x, t)
$$

with periodic boundary conditions

$$
u_{\alpha}(\boldsymbol{x}, t+2 \pi / \omega)=u_{\alpha}(\boldsymbol{x}, t)
$$

It is of central importance to note that the solutions of equation (2.3) fall into equivalence classes, which means that for a fixed value of $n$ and any integer $m$ the expressions

$$
\begin{aligned}
& u_{\alpha}(\boldsymbol{x}, t)=u_{n}(\boldsymbol{x}, t) \exp (\mathrm{i} m \omega t) \\
& \varepsilon_{\alpha}=\varepsilon_{n}+m \omega
\end{aligned}
$$

represent the same Floquet state (2.2); we use the symbol $\alpha$ for the double index

$$
\alpha=(n, m)
$$

and employ the convention to denote by $u_{n}(x, t) \equiv u_{(n, 0)}(x, t)$ the representative that originates from the time-independent $H_{0}$ eigenfunction labelled by the quantum number $n$.

The physical relevance of the Floquet states stems from the fact that for sufficiently slow time variation of external parameters, e.g. $\lambda=\lambda(t)$, the adiabatic solution of Schrödinger's equation evolves on quasi-energy surfaces in parameter space: a wavefunction initially in a Floquet state remains in the connected Floquet state at each moment of time.

In a strict sense, the validity of the adiabatic theorem is questionable, since, due to the class structure (2.5), the quasi-energy spectrum is (in general) dense on the real axis and the state under consideration is never separated by a spectral gap from the other states, a necessary assumption for a rigorous proof of the adiabatic theorem (Born and Fock 1928, Avron et al 1987).

For a given experimental apparatus, however, effective, smooth quasi-energy surfaces can be constructed (Breuer et al 1989), on which an adiabatic theorem holds. In general, deviations from adiabatic behaviour can be traced to near-degeneracies (avoided level crossings (AC) (Breuer et al 1988)). Assuming an isolated avoided crossing located at the field strength $\lambda_{c}$ in an $\varepsilon-\lambda$ plot, we obtain for the probability of a diabatic transition (Breuer and Holthaus 1989).

$$
P=\exp \left(-\frac{1}{2} \pi \eta\right)
$$

where

$$
\eta=\left.(\delta \varepsilon \delta \lambda / \dot{\lambda})\right|_{\lambda_{\dot{c}}}
$$

Here $\delta \varepsilon$ is the minimal separation of the anticrossing quasi-energy levels in one Brillouin zone and $\delta \lambda$ denotes the $\lambda$ interval in which this distance increases by a factor of $\sqrt{ } 2$. Now the probability (2.6) is almost equal to 1 for narrow AC and/or rapid parameter variation (i.e. $\dot{\lambda}$ large), which means that the wavefunction 'jumps' over the $\mathrm{AC}$ without changing, i.e. it behaves as if the anticrossing level were not there at all. Therefore, such a narrow AC can be neglected. Actually, the AC lie dense since there is one representative of every quasi-energy class (2.5) in each Brillouin zone and, according 
to the von Neumann-Wigner theorem (Von Neumann and Wigner 1929), all eigenvalues within the same symmetry class repel each other. Disregarding all AC below a certain scale corresponds to a coarse-graining procedure by which a few, dynamically relevant, levels are selected from a dense set of quasi-energy eigenvalues; these form smooth 'effective' quasi-energy surfaces separated by well defined gaps (Breuer et al 1989).

It should be noted that $\delta \lambda / \dot{\lambda}=: \Delta \tau$ can be interpreted as the time during which the wavefunction feels the influence of the $A C$. Thus it depends on the timescales set by experiment whether or not an $\mathrm{AC}$ can be omitted in the coarse-graining procedure.

In the following, we shall apply this general procedure to describe hydrogen atoms exposed to strong microwave fields.

A full numerical analysis of (2.1) in three dimensions is not yet feasible; however, it has been convincingly demonstrated that a $(1+1)$-dimensional model (Jensen 1982, 1984)

$$
\left(H_{0}+\lambda x \sin \omega t-\mathrm{i} \partial_{t}\right) \psi(x, t)=0
$$

with a static Hamiltonian

$$
H_{0}=\frac{1}{2} p^{2}+V(x)
$$

where

$$
V(x)= \begin{cases}-1 / x & x>0 \\ +\infty & x \leqslant 0\end{cases}
$$

already yields a reasonably good description of the microwave experiments in question. We denote the unperturbed eigenstates of $H_{0}$ by $\varphi_{n}(x)$, i.e.

$$
H_{0} \varphi_{n}(x)=E_{n} \varphi_{n}(x) \quad E_{n}=-1 /\left(2 n^{2}\right) .
$$

Owing to the presence of the continuous spectrum of (2.9), the eigenvalues of $H_{0}$ turn into resonance poles on the second Riemannian sheet of the complex $\varepsilon$ plane for non-vanishing field strength $\lambda$ (Yajima 1982). The quasi-energies obtain a negative imaginary part, which reflects the finite lifetime of the Floquet states. However, it has been shown that the model (2.9), (2.10) reproduces experimental data even when restricted to the space spanned by the bound states; in this approximation the quasienergies are real.

Since we are interested in the adiabatic evolution of bound states, we also employ this approximation; the following numerical calculations were performed in the space spanned by the bound states $\left\{\varphi_{n} \mid n=25, \ldots, 105\right\}$. We set $\omega=1.508 \times 10^{-6}$ au corresponding to a frequency of $9.92 \mathrm{GHz}$, which has been used in the experiments reported by Jensen et al (1988).

First of all, in the experiments on microwave ionisation a slow variation of the field strength is caused by the fringe fields, which the atoms must cross when entering the microwave cavity. To investigate the effect of the fringe fields on different initial states, we model this situation by a smoothly increasing amplitude

$$
\lambda(t)= \begin{cases}\lambda_{\mathrm{f}} \exp \left(-t^{2} / 2 T_{\mathrm{a}}^{2}\right) & t \leqslant 0 \\ \lambda_{\mathrm{f}} & t>0\end{cases}
$$

and solve the Schrödinger equation (2.8) with initial condition

$$
\psi(x, t) \rightarrow \varphi_{n_{0}}(x) \exp \left(-\mathrm{i} E_{n_{0}} t\right) \quad \text { for } t \rightarrow-\infty .
$$


When the final field strength is reached $(t=0)$, we decompose the wavefunction $\psi(x, t=0)$ into the Floquet states corresponding to the amplitude $\lambda_{\mathrm{f}}$ and calculate the probabilities

$$
\left|a_{n_{0}}\right|^{2}:=\left|\left\langle u_{n_{0}}(x, t=0) \mid \psi(x, t=0)\right\rangle\right|^{2}
$$

as a function of the switch-on time $T_{\mathrm{a}}$. For each value of $n_{0}, \lambda_{\mathrm{f}}$ is chosen such that the quasi-energy level originating from $E_{n_{0}}$ undergoes only completely negligible $\mathrm{AC}$ in the interval $\left[0, \ldots, \lambda_{\mathrm{f}}\right]$, where the term negligible refers to $\mathrm{AC}$ unnoticed in the sense of the coarse-graining procedure proposed above. Thus, the Floquet state $u_{n_{0}}$ that effectively develops from the stationary eigenstate $\varphi_{n_{0}}$ can be unambiguously identified.

In figure 1 we plot the results for some values of $n_{0}$ from $n_{0}=38$ to $n_{0}=73$ as a function of $T_{\mathrm{a}} ; T_{\mathrm{a}}=0$ means sudden turn-on of the field and $\left|a_{n_{0}}\right|^{2} \approx 1$ indicates that the effective adiabatic limit has been reached. Note that the scaled field strength $n_{0}^{4} \lambda_{\mathrm{r}}$ is successively decreased. The adiabatic behaviour is obvious: even if the parameter

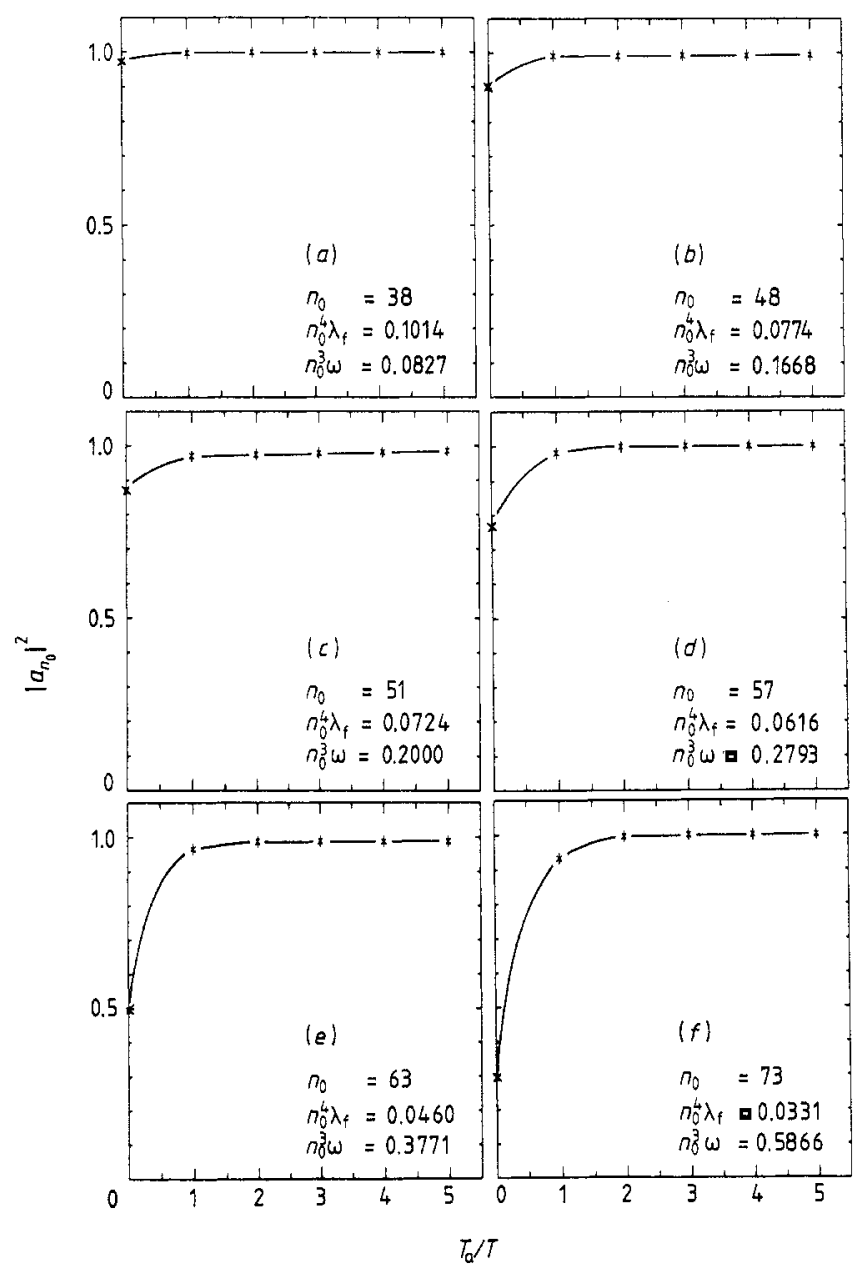

Figure 1. Occupation probability of the Floquet state connected to the initial stationary state $n_{0}$ as a function of the switch-on time $T_{a}$ measured in periods of the external field (cf. equation (2.14)). In all cases $\omega=1.508 \times 10^{-6}$ au. 
variation is limited to only a few cycles of the external field, it suffices to shift the initial state into the continuously connected Floquet state.

Whereas, for scaled frequencies greater than 0.3 , this effect is extremely important (figures $1(e)$ and $(f)$, we encounter for very low frequencies $n_{0}^{3} \omega<0.1$ the traditional adiabatic behaviour (figure $1(a)$ ). Here, the external field changes so slowly compared to the period of the Kepler orbital motion that the wavefunction follows instantaneous Stark states. A Floquet state for a fixed moment $t_{0}$ is approximately given by

$$
u_{n_{0}}\left(x, t_{0}\right) \simeq \varphi_{n_{0}}^{\text {Stark }}\left(x, \lambda \sin \omega t_{0}\right)
$$

where the right-hand side denotes the eigenfunction of the Stark Hamiltonian

$$
H_{0}+\lambda x \sin \omega t_{0} \quad t_{0} \text { fixed. }
$$

Thus we have for integer multiples of the external field period $T$

$$
u_{n_{0}}(x, k T) \approx \varphi_{n_{0}}^{\text {Stark }}(x, 0)=\varphi_{n_{0}}(x) \quad n_{0}^{3} \omega<0.1
$$

and therefore from $(2.14)\left|a_{n_{0}}\right|^{2} \approx 1$ independent of $T_{\mathrm{a}}$.

Hence in the low-frequency regime we have the correspondence

$$
\left\{\text { family of Stark eigenstates for } t_{0} \in[0, T]\right\} \Leftrightarrow 1 \text { Floquet state }
$$

and the energies $E_{n_{0}}$ of this one-parameter family of Stark states and the Floquet index $\varepsilon_{n_{0}}$ are related by

$$
(1 / T) \int_{0}^{T} \mathrm{~d} t_{0} E_{n_{0}}\left(\lambda_{\mathrm{f}} \sin \omega t_{0}\right) \simeq \varepsilon_{n_{0}}\left(\lambda_{\mathrm{f}}\right) .
$$

As figure 1(a) shows, (2.18) may hold even for relatively large values of $n_{0}^{4} \lambda_{f}$; indeed this correspondence can be used for a viable treatment of the low-frequency dynamics (Richards 1987). We emphasise, however, that an efficient description in terms of adiabatic evolution in an effective space of Floquet states is viable for all scaled frequencies, the 'adiabatic' Stark picture appearing only as the limiting case in the $n_{0}^{3} \omega<0.1$ regime.

\section{Non-monotonic structures in the ionisation signal}

We now turn to the relationship between properties of quasi-energy surfaces and experimentally observed ionisation probabilities. To begin with we plot in figure 2 one Brillouin zone of Floquet indices originating from states with principal quantum numbers $n$ from 36 to 40 as a function of the field strength $\lambda$ in an experimentally interesting region. (Numerically, this can be achieved by selecting from all the eigenvalues of the one-cycle propagator those whose corresponding eigenstates have their main support in the space spanned by $\left\{\varphi_{n} \mid n=36, \ldots, 40\right\}$.)

We observe a large dynamic Stark shift: the quasi-energies decrease monotonically over several Brillouin zones without showing, on the scale imposed in figure 2, visible avoided crossings. We note, however, that several levels come close to each other in the interval of field strength marked in figure 2 . If we now look at this interval on a finer scale (figure 3 ), we see that the levels of figure 2 actually respond very differently to the external field: whereas the state $n=36$ still looks completely intact, i.e. does not show any AC on the scale of figure 3 , level $n=38$ is broken by more and more narrow $A C$, and at the same time it is crossed by the remnants of the higher states that can no 


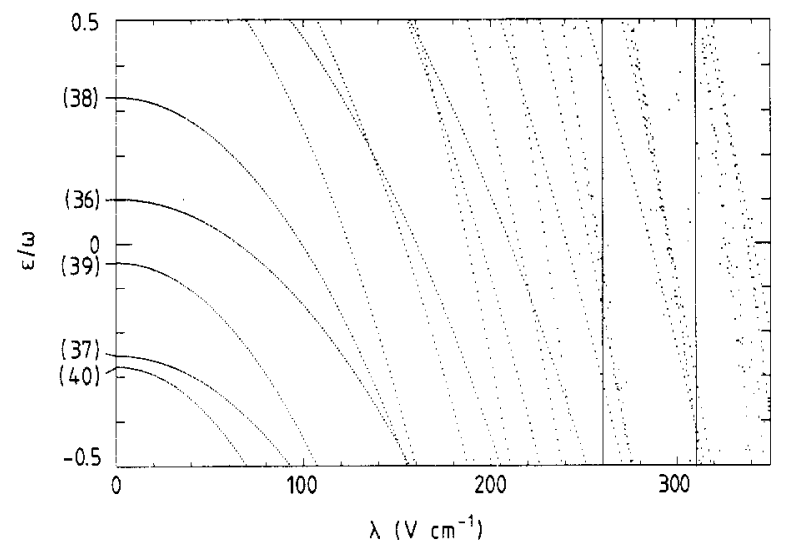

Figure 2. One Brillouin zone of quasi-energies of the $(1+1)$-dimensional hydrogen atom (2.8), (2.9) as a function of the field strength. Integers in parentheses denote principal quantum numbers. The marked area is shown enlarged in figure 3.

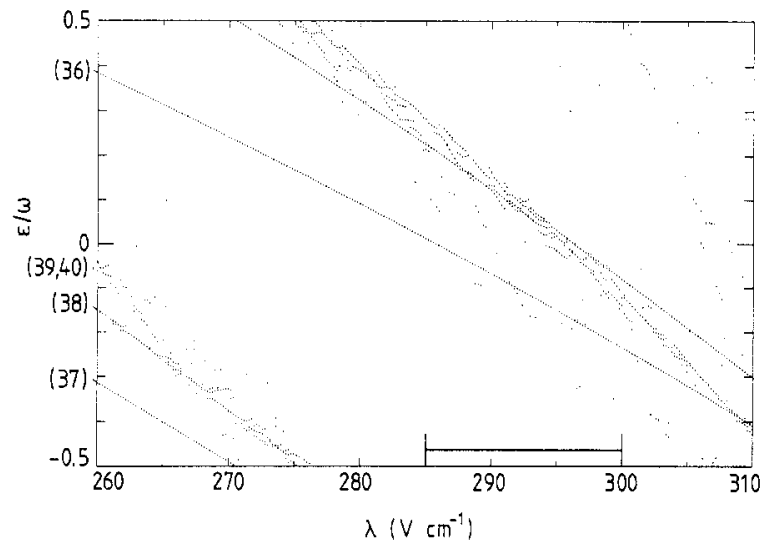

Figure 3. Enlarged section of the spectrum in figure 2. The marked interval of field strength indicates the 'critical region'.

longer be identified as individual states by any reasonable means of identifying quantum numbers. A particularly interesting feature is that the level $n=37$ is seen to undergo a 'hyper-avoided crossing' with this group of states in the region of field strength that is marked in figure 3 and which will be denoted 'critical region' hereafter.

Such spectral properties should lead to experimentally observable consequences. As we emphasised elsewhere (Breuer and Holthaus 1989), the adiabatic behaviour breaks down when too many 'relevant' AC appear: for example, Landau-Zener type transitions in the fringe field will lead to the population of fast ionising Floquet states. Generally, the appearance of multiple avoided crossings above a certain scale indicates strong mixing with higher-lying and, hence, fast-delocalising states.

Indeed, it is found experimentally (Jensen et al 1988) that the intact level $n=36$ does not significantly ionise in the whole region of field strength shown in figure 3 whereas $n=39$ and higher states start to ionise at lower values. Furthermore, the ionisation signal for atoms initially prepared in the states $n=37,38$ shows a nonmonotonic behaviour as a function of the field strength, i.e. a 'bump', at approximately 
$\lambda \simeq 300 \mathrm{~V} \mathrm{~cm}^{-1}$. Such a phenomenon can be qualitatively understood from the structure of the quasi-energy spectrum, as we now show using the example of $n=37$.

(i) First, assume a cavity field strength below the critical region of figure 3 , e.g. $\lambda_{\mathrm{f}}=270 \mathrm{~V} \mathrm{~cm}^{-1}$. The atom responds purely adiabatically and is, when entering the cavity, shifted into the connected Floquet state, remains stable and is shifted back when leaving the cavity.

(ii) Next, assume a final field strength above the critical region, e.g. $\lambda_{\mathrm{f}}=320 \mathrm{~V} \mathrm{~cm}^{-1}$. Now the state has to cross the critical region in the fringe field. However, all $\mathrm{AC}$ encountered are so sharp that the probability (2.6) at each of them is very close to 1 . Stated differently, when passing the critical region in the cavity fringe fields the system does not see the AC long enough to resolve them. Hence in this situation we are entitled to neglect all the avoided crossings in the critical region and to deal with one effective state $n=37$. This is an explicit example of the coarse-graining procedure and effective adiabatic motion.

(iii) The situation changes if the final field strength is just in the critical region and stays approximately constant over several hundred cycles of the microwave field. This means that the interaction time is long enough to resolve the narrow quasi-energy gaps of the avoided crossings and to cause transitions into the group of states $n=38$, 39,40 , which are more unstable and tend to ionise.

Hence we see the experimental 'bumps' to be related to such a critical crossing region.

To make this statement more definite we solved the Schrödinger equation for 300 cycles of the external field with the initial condition $\psi(t=0)=\varphi_{37}$. Now, the explicit modelling of the fringe field is not necessary, since for $n=37$ we have the low-frequency correspondence $(2.17),(2.18)$ and the initial state is shifted into the correct quasi-energy state anyway (see figure $1(a)$ for $n=38$; it is clear that $(2.17$ ) holds even better for $n=37)$.

Obviously a model that excludes the continuum is not sufficient to give a complete description of ionisation experiments. However, we are mainly interested in qualitative mechanisms giving rise to non-monotonic behaviour of the ionisation signal, which should be due to the bound-state dynamics (Blümel and Smilansky 1987, Jensen et al 1988). Since it is reasonable to assume that Floquet states ionise more rapidly if they contain more high-lying eigenstates of $H_{0}$, we plot $P_{\text {ion }},\left\langle b_{36}\right\rangle$ and $\left\langle b_{38}\right\rangle$ in figure $4(b)$, where we introduced, as a rough measure for the ionisation probability $\dagger$,

$$
P_{\text {ion }}=\sum_{n \geq 39}\left\langle b_{n}\right\rangle
$$

and

$$
\left\langle b_{n}\right\rangle=(1 / 300) \sum_{m=0}^{300}\left|\left\langle\varphi_{n} \mid \psi(t=m T)\right\rangle\right|^{2}
$$

We have checked that de-excitation to lower states is of minor importance. A juxtaposition of the spectrum in figure $4(a)$ with figure $4(b)$ clearly confirms our explanation: right below the critical crossing region a bump appears.

Similar calculations leading to a bump structure have been reported by Blümel and Smilansky (1987) who linked it to 'unresolved clusters' of avoided crossings. In the light of our findings we are now in a position to specify this notion in a more precise

\footnotetext{
† We use time averaging to avoid distinguishing a fixed moment of time.
} 


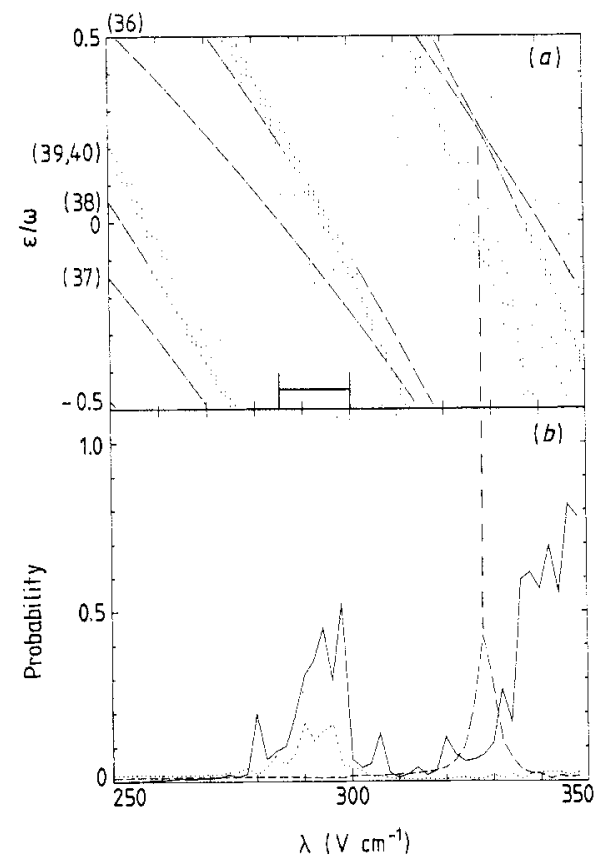

Figure 4. Comparison of a part of the coarse-grained quasi-energy spectrum $(a)$ with the numerically calculated ionisation signal $(b)$ for the initial state $n_{0}=37$. Full curve, $P_{\text {ion }}$; broken curve, $\left\langle b_{36}\right\rangle$; dotted curve, $\left\langle b_{38}\right\rangle$ (cf. equations (3.1) and (3.2)). Note that the position of the bump coincides with the position of the critical region. Furthermore an isolated AC between $n=37$ and $n=36$ produces a pronounced de-excitation profile.

manner. As we have discussed previously (Breuer et al 1989), the set of unresolved $\mathrm{AC}$ is, in general, dense. Notwithstanding this fact, our calculations lead us to the following interpretation.

(i) Only a few Floquet states are involved in the mechanism producing the bump structure (figure 3). When they are nearly degenerate, they 'mix', showing many AC.

(ii) A coarse-graining procedure yields effective quasi-energy surfaces that can be connected to the static eigenvalues of $H_{0}$ (figure 2). Since the wavefunction, when entering the cavity, follows these surfaces, this allows for a clear identification of the relevant Floquet states.

A very similar analysis as presented above for $n=37$ can be performed for $n=38$; our numerical result for the corresponding ionisation curve is very similar to the one published by Blümel and Smilansky (1987). However, the aim of our present investigation is not to reproduce experimental data by numerical simulations but to explain structures in the ionisation signal by structures in the quasi-energy spectrum: figure 2 demonstrates a near-degeneracy of the coarse-grained $n=38$ level with higher states and with $n=37$ at $\lambda \simeq 300 \mathrm{~V} \mathrm{~cm}^{-1}$, which is indeed the correct position for the most prominent 'bump' found experimentally.

As has been noted by Blümel and Smilansky (1987), the individual spikes in the numerical ionisation signal are due to individual avoided crossings too close to be resolved experimentally. However, figure 4 indicates that it should be possible to observe the effect of isolated AC: our model exhibits a well separated AC between states $n=36$ and $n=37$ at a field strength of about $\lambda \simeq 330 \mathrm{~V} \mathrm{~cm}^{-1}$ and as a consequence the 
solution of the Schrödinger equation shows a strong de-excitation $n=37 \rightarrow n=36$. Now, the underlying physical mechanism is not a Landau-Zener transition (2.6), i.e. a full passage through the $\mathrm{AC}$, but instead the variation of the field strength stops within the region of the AC. An analytical expression for the transition probability can be obtained from a two-Floquet-state calculation. Considering two Floquet states $i$ and $f$ undergoing an isolated $\mathrm{AC}$ at $\lambda=\lambda_{\mathrm{c}}$ and using the Floquet Hamiltonian (Shirley 1965)

$$
\mathscr{H}=\frac{\delta \varepsilon}{2}\left(\begin{array}{cc}
\left(\lambda-\lambda_{c}\right) / \delta \lambda & 1 \\
1 & -\left(\lambda-\lambda_{c}\right) / \delta \lambda
\end{array}\right)
$$

$(\delta \varepsilon, \delta \lambda$ are defined as in (2.7)) we solve the Schrödinger equation in the extended Hilbert space $\mathscr{H} \Phi=\mathrm{i} \partial_{\tau} \Phi$ (Breuer and Holthaus 1989) and finally arrive at

$$
P_{i \rightarrow f}(\lambda) \simeq \frac{1}{2} \frac{1}{1+\left[\left(\lambda-\lambda_{\mathrm{c}}\right) / \delta \lambda\right]^{2}} .
$$

Owing to the finite width of the $A C$ also the (de-)excitation profile obtains a finite width. For $i=37$ and $f=36$ this formula agrees well with the numerically computed curve in figure $4(b)$; the value of $\delta \lambda$ can be determined from figure $4(a)$. It should be clear that the resolution of such an $\mathrm{AC}$ is a long-time effect (cf. figure 7 in Breuer et al 1988); $\delta \varepsilon$ has dropped out of (3.4). Even the AC of figure $4(a)$ yields a diabatic transition probability of $P \simeq 0.93$, when, for example, the external field rises from 0 to $350 \mathrm{~V} \mathrm{~cm}^{-1}$ over a period of 80 cycles.

\section{Conclusions}

The Floquet picture yields an intuitively clear way of understanding the low-frequency ionisation of hydrogen atoms. For a given experimental set-up with its specific resolution power an effective (finite-dimensional) set of Floquet states can be constructed which leads to effective smooth quasi-energy surfaces allowing for the location of $A C$ and an identification of the states involved in a particular process. Adiabatic motion on these surfaces is a well defined concept.

The feature that we examined here is the low-frequency bump structure, which we found to originate from a near-degeneracy of a few effective Floquet states.

In a way, our coarse-graining procedure provides a link between the "unresolved clusters' of Blümel and Smilansky (1987) and the approach of Jensen et al (1988) where the existence of non-monotonic structures was related to resonances between a few 'adiabatic states' (LHS of (2.18)): quasi-energy surfaces originally broken by many AC can be smoothed out to yield a few effective surfaces (Breuer et al 1989).

Finally we should like to point out some experimental implications. First, we re-emphasise that it depends on the experimental timescales to decide which $\mathrm{AC}$ are resolved and which are not; different devices will, in general, lead to different results. Secondly, it should be possible to experiment with isolated avoided crossings: peaked structures in the transition cross section like the one we found for the de-excitation $n=37 \rightarrow n=36$ should not be confounded with resonances in the classic sense. The de-excitation peak can also be observed in the excitation spectrum of $n=36$ Rydberg atoms: the $n=37$ level should be seen with large probability and a pumping $n=36 \rightarrow n=$ 37 with a ' 13.6 photon transition' (a misleading term indeed) with maximal probability $\frac{1}{2}$ would have been achieved. Phenomena like this are pure bound-state effects and 
are in principle detectable by measuring the population of individual bound states after the atoms have left the microwave cavity.

\section{Acknowledgment}

We would like to thank the 'Höchstleistungsrechenzentrum' at the KFA Jülich for a grant of computation time on the CRAY X-MP/416.

\section{References}

Avron J E, Seiler R and Yaffe L G 1987 Commun. Math. Phys. 11033

Bayfield J E and Koch P M 1974 Phys. Rev. Lett. 33258

Blümel R and Smilansky U 1987 Z. Phys. D 683

Born M and Fock V 1928 Z. Phys. 51165

Breuer H P, Dietz K and Holthaus M 1988 Z. Phys. D 1013

- 1989 Preprint AM-89-01, Bonn

Breuer H P and Holthaus M 1989 Z. Phys. D 111

Casati G, Chirikov B V, Shepelyanski D L and Guarneri I 1987 Phys. Rep. 15477

Jensen R V 1982 Phys. Rev. Lett. 491365

1984 Phys. Rev. A 30368

Jensen R V, Koch P M, Galvez E J, van Leeuwen K A H, Moorman L, Sauer B E, Leopold J G and Richards D 1988 Preprint; J. Phys. B: At. Mol. Opt. Phys. submitted

Richards D 1987 J. Phys. B: At. Mol. Phys. 202171

Shirley J H 1965 Phys. Rev. 138 B979

Von Neumann J and Wigner E 1929 Phys. Z. 30467

Yajima K 1982 Commun. Math. Phys. 87331

Zel'dovich Ya B 1967 Sov. Phys.-JETP 241006 